\title{
An Anti-jamming Algorithm for Attitude Measurement Based on Antenna Array
}

\author{
Jian Hua CUI ${ }^{1, a}$, Nai Ping CHENG ${ }^{1}$ and Pan Feng HE ${ }^{1}$ \\ ${ }^{1}$ Department of Optoelectronic Equipment, Academy of Equipment, 101416 Huairou District Yanqi Town, Beijing
}

\begin{abstract}
Aiming at the problems existing in the attitude measurement using carrier phase of navigation signal in the jamming environment, an antenna array for anti-jamming attitude measurement is constructed. The antenna array is constructed by three circle array with core. An anti-jamming measurement method based on antenna array is proposed. This method uses array antenna to estimate the direction of arrival jamming signal though MUSIC algorithm. Then the adaptive nulling algorithm based on the linear constrained minimum variance algorithm is used to eliminate the jamming and hold the navigation signal. Finally, the double difference method is used to measure the attitude. The whole attitude information is obtained by the attitude measurement of the two baselines. Simulation has been made for eliminating the jamming with adaptive nulling algorithm. Comparison of the attitude measurement accuracy is also made by simulation. The results show that the algorithm we proposed can significantly inhibit the jamming and improve the accuracy of attitude measurement.
\end{abstract}

\section{Introduction}

Attitude measurement though the carrier phase of the navigation satellite signal is an important part of the satellite navigation application. The attitude measurement system based on GPS has high precision, whose initialization time is relatively short. The system also has high stability. Compared with the traditional way of measuring attitude, it has its own advantages.

Attitude measurement system is of great significance in Aeronautics and Astronautics, and is widely used in various kinds of aircraft and ships. But because the accuracy of the position measurement depends on the carrier phase of the navigation signal, it needs to solve the integer ambiguity in the attitude calculation process, and the operation is larger. The measuring accuracy is affected by the baseline length of the measuring antenna too. Under the condition of modern war, the navigation signal is very vulnerable to various kinds of interference, so that the accuracy of attitude measurement algorithm is reduced. Even more, the attitude measurement algorithm is invalid.

At present, there are many applications and researches on the adaptive nulling antenna[1,2], but there are few researches on the application of the adaptive control algorithm in the attitude measurement. The antiinterference attitude measurement method is based on the antenna array to form an adaptive nulling antenna. The signal of each channel is weighted by amplitude and phase under certain constraint conditions, which forms a null in the direction of the interference signal. Then the synthesized signal is used for attitude measurement. At the same time, due to the difference of the attitude measurement antenna, we construct an antenna array which is suitable for anti-interference attitude measurement. The output of each measuring antenna is a signal after eliminating interference.

This method has certain effect for the attitude measurement based on carrier phase measurement, but there are also some problems. The reason is that in the amplitude and phase weighting process, the signal phase information of each array element received is destroyed. The carrier phase exists error. Through this way to measure the attitude can improve the accuracy in the interference environment.

As the anti-jamming attitude measurement algorithm and anti-jamming algorithms differ in the constraint conditions, so they should have different constraint criterion[3]. The constraint criterion used in anti-jamming algorithm is also not suitable for anti-jamming attitude determination algorithm. For this purpose, we choose the Linearly Constrained Minimum Variance (LCMV) as the optimization criterion of the nulling antenna. In this way, it can ensure the correct measurement of the carrier phase in the interference condition, and ensure the accurate measurement of the attitude.

\section{Signal model}

In order to realize anti-jamming attitude measurement on the carrier, the right antenna arrays are needed. There are many types of antenna arrays, such as linear array, planar array, uniform circular array, circular array with core, etc.

\footnotetext{
a Corresponding author: cjh_xilinx@163.com
} 
Anti-jamming attitude measurement algorithm is realized based on a 7 element circular array measuring antenna with core. The middle element is the reference element. Studies have shown that in nulling antenna, the null can be generated effectively in the circular array with core, a flat pattern can be generated in the expected direction. These features meet the requirements of the array antenna, so we use a circular array with a core as the measuring antenna array. The antenna structure is shown in Fig. 1:

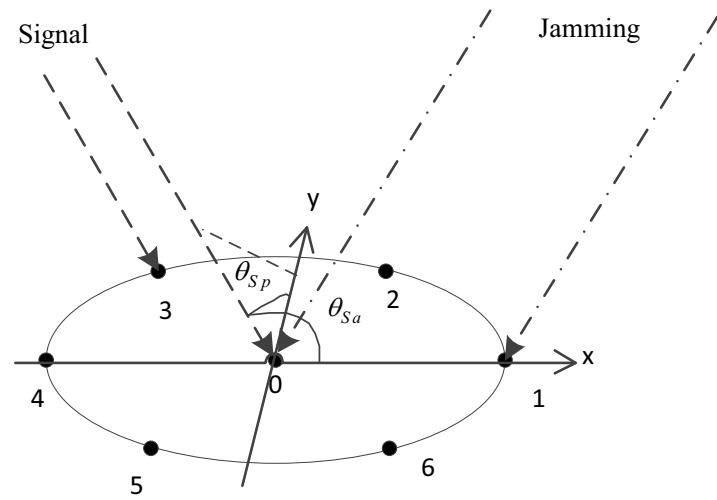

Figure 1. Formatting sections, subsections.

Element 0 is the reference, a circular array is formed by the element 1-6 which is uniformly distributed on the circumference. The $\mathrm{X}$ axis is established by the array element 4-0-1, the $Y$ axis is perpendicular to the $X$ axis in the antenna plane. The $Z$ axis is perpendicular to the plane of the antenna. The vector coordinate system is established, and the position of the array element 0 is the coordinate origin. Each array element spacing is the base line length $l_{b}$, the signal carrier wave length is $\lambda$. In order to do not have the fuzzy degree, we take $l_{b} \leq \lambda / 2$.

For the 7 array elements antenna with a core, compared with the reference array element 0 , the angle of the element $i$ relative to the positive $\mathrm{X}$ axis is: $\theta_{i}=(i-1) \pi / 3$. For a signal with an random angle $\left(\theta_{p}, \theta_{a}\right)$, the phase delay of the array element $i$ and the reference array element can be expressed as:

$$
\Delta \theta_{S i}=2 \pi l_{b} \sin \left(\theta_{S p}\right)\left[\cos \left(\theta_{S a}-\theta_{i}\right)\right] / \lambda
$$

So the direction vector is:

$$
D=\left(1, e^{\Delta \theta_{S 1}}, e^{\Delta \theta_{S 2}}, \ldots, e^{\Delta \theta_{S 6}}\right)
$$

where $\Delta \theta_{S i}$ is the phase delay.

\section{Anti-jamming attitude measurement method based on array antenna}

Our method for anti-jamming attitude measurement is composed of three parts, first of all need to direction of arrival estimation of signal to interference, arriving direction of the interference signals need to be estimated. After DOA (Direction Of Arrival) estimation, equations are established based on the geometric relationship between wave direction and antenna array. The optimal weight vector is solved according to the established equations and constraints. The array output signal after the interference suppression is calculated based on the weight vector and the antenna input. Finally, the attitude of the carrier is measured according to the output signal of the three antenna array. This is the whole process of anti-jamming attitude measurement.

\subsection{DOA estimation of the jamming}

In the processing of anti-jamming attitude measurement, in order to eliminate the interference, the DOA of the interference signal need to be estimated, interference is eliminated using the wave direction information to phase shift. In $\mathrm{N}$ element antenna arrays, N-1 interference can be eliminated. We use the traditional MUSIC method to measure the DOA of the interference. The interference direction is obtained by using the orthogonality of the interference signal and the noise signal in the characteristic vector. The specific process includes the following parts:

1 According to the input signal of each array element at different time $X_{i}=\left(x_{0}, x_{1}, \ldots x_{7}\right)_{i}$, the covariance matrix of the input signal of each array element of the antenna array is calculated:

$$
\hat{R}_{X}=\frac{1}{N} \sum_{i=0}^{N-1} X_{i} X_{i}^{H}
$$

2 After the establishment of covariance matrix, eigenvalues and eigenvectors are obtained by means of eigenvalue decomposition of $\hat{R}_{X}$ :

$$
\hat{R}_{X} V=V \Lambda
$$

where $\Lambda=\operatorname{diag}\left\{\lambda_{0}, \lambda_{1}, \ldots, \lambda_{N-1}\right\}, \lambda_{0} \geq \lambda_{1} \geq \ldots \geq \lambda_{N-1}$ is the eigenvalues, and $V=\left[p_{0}, p_{1}, \ldots, p_{N-1}\right]$ is the eigenvectors matrix. The eigenvectors of the covariance matrix contains two parts, the signal subspace with larger values and the noise subspace with smaller values. Direction vector of the interfering signals belongs to the signal subspace, and is orthogonal to the noise subspace.

3 In the input covariance matrix, all the eigenvalues of the noise are relatively small. If the number is $\mathrm{M}$, the corresponding interference number is $\mathrm{K}=\mathrm{N}-\mathrm{M}$. The characteristic vector of the signal is orthogonal to the characteristic vector of the noise. Constructing the noise subspace by using the characteristic vector corresponding to the eigenvalues of the $\mathrm{M}$ :

$$
V_{n}=\left[p_{K}, p_{K+1}, \ldots, p_{N-1}\right]
$$

4 Structure MUSIC spectra for the incident signal:

$$
P_{\text {MUSIC }}=\frac{1}{D^{H} V_{n} V_{n}^{H} D}
$$

The characteristic vector of the interference signal and the noise is orthogonal, $D^{H} V_{n} V_{n}^{H} D$ should be zero. That is to say, at the peak of the MUSIC spectrum, the direction vector is corresponding to the direction of arrival. If at this time the corresponding peak of the incident angle is $\left(\theta_{p}, \theta_{a}\right)$, the incident angle is the direction of the incoming wave.

\subsection{The adaptive nulling based on antenna array}

In the navigation system, the GPS navigation signal will be transmitted after the pseudo code spread spectrum, and the basic signal model received by the reference antenna in the receiving antenna is $S=s P e^{-j\left(\omega_{c} t+\varphi_{S 0}\right)}$, 
where $P$ is the spreading pseudo code, $s$ is the original signal, $\omega_{c}$ is the carrier angular velocity, $\varphi_{s 0}$ is the initial phase.

For the center reference array element of the array antenna, hypothesis in the process of signal propagation, the number of jamming is $\mathrm{M}-1$. The jamming is $J_{i}=j_{i} e^{-j\left(\omega_{c} t+\varphi_{J_{i}}^{0}\right)}(i=1 \ldots M-1)[4]$, at the same time the environmental noise is $N_{0}$, the signal received by the central reference array is:

$$
R_{0}=\left(\begin{array}{llll}
1 & 1 & \ldots & 1
\end{array}\right)\left(\begin{array}{llll}
S & J_{1} & \ldots & J_{\mathrm{M}-1}
\end{array}\right)^{T}+N_{0}
$$

As shown in Fig.1, in the seven array element antenna array, azimuth is defined as the angle between the signal and the $\mathrm{X}$ axis, pitch angle is defined as the angle between the signal and the array antenna. The baseline length between each array element and the reference array is $l_{b}$, carrier wave length is $\lambda$. For arbitrary array elements, the incident azimuth angle of the signal is $\theta_{S p}$, the pitch angle is $\theta_{S a}$, the incident azimuth angle of the jamming is $\theta_{J_{i} p}$, the pitch angle is $\theta_{J_{i} a}$. If the angle between the array element $k$ and the $\mathrm{X}$ axis is $\theta_{k}(k=1 \ldots$ 6 ), for any array element $k$, relative to the reference array, the carrier phase difference of the received signal is:

$$
\Delta \theta_{S_{k}}=2 \pi l_{b} \sin \left(\theta_{S p}\right)\left[\cos \left(\theta_{S a}-\theta_{k}\right)\right] / \lambda
$$

The carrier phase difference for jamming $i$ received is:

$$
\Delta \theta_{J_{i}^{k}}=2 \pi l_{b} \sin \left(\theta_{J_{i} p}\right)\left[\cos \left(\theta_{J_{i} a}-\theta_{k}\right)\right] / \lambda
$$

Therefore, the signal model of any array element $K$ received is:

$$
\left.\begin{array}{l}
R_{k}=\left(\begin{array}{lll}
e^{j \Delta \theta_{S_{k}}} & e^{j \Delta \theta_{J_{1}^{k}}} \quad \ldots \quad e^{j \Delta \theta_{J_{M-1}^{k}}}
\end{array}\right) \\
\left(\begin{array}{llll}
S & J_{1} & \ldots & J_{M-1}
\end{array}\right)^{T}+N_{k} \quad(M=1 \ldots 6
\end{array}\right)
$$

The model of the received signal of all the elements can be used to form the equation:

$$
\begin{aligned}
& \left(\begin{array}{llll}
R_{0} & R_{1} & \ldots & R_{M-1}
\end{array}\right)^{T}=A\left(\begin{array}{lllll}
S & J_{1} & \ldots & J_{M-1}
\end{array}\right)^{T} \\
& +\left(\begin{array}{llll}
N_{0} & N_{1} & \ldots & N_{M}
\end{array}\right)^{T} \quad(M=6) \\
& A=\left(\begin{array}{cccc}
1 & 1 & \ldots & 1 \\
e^{j \Delta \theta_{S_{1}}} & e^{j \Delta \theta_{J_{1}^{1}}} & \ldots & e^{j \Delta \theta_{J_{N-1}^{1}}} \\
\mathrm{M} & \mathrm{M} & \mathrm{O} & \mathrm{M} \\
e^{j \Delta \theta_{S_{N-1}}} & e^{j \Delta \theta_{J_{1}^{N-1}}} & \ldots & e^{j \Delta \theta_{J_{N-1}^{N-1}}}
\end{array}\right)
\end{aligned}
$$

Since our main application scenario is to measure the attitude, we need to find the signal's phase as far as possible without distortion. So in the interference environment, the linearly constrained to minimum variance(LCMV) can make signal output power minimized with the guaranteed gain in the signal direction[5], which can try to suppress the interference maximum, while maintaining signal produces less distortion.

According to the LCMV criterion, the optimal weights are generated:

$$
\omega_{\text {opt }}=\hat{R}_{X}^{-1} A\left(A^{H} \hat{R}_{X}^{-1} A\right)^{-1} g
$$

where $g=(1, \ldots, 1,0, \ldots, 0)$, the number of ' 1 ' is the number of the signals, the number of ' 0 ' is the number of the jammings.

Under the influence of the optimal weight, the output after nulling is getting from:

$$
Y=\omega X
$$

For the anti-jamming attitude determination, the output of each antenna is supposed to be the signal after the antijamming algorithm, because this is the only way to ensure that the distortion of the carrier phase is minimized. Therefore, in the application of attitude measurement, the three measurement antennas should be in the form of anti-jamming antenna array, the structure is shown as below:

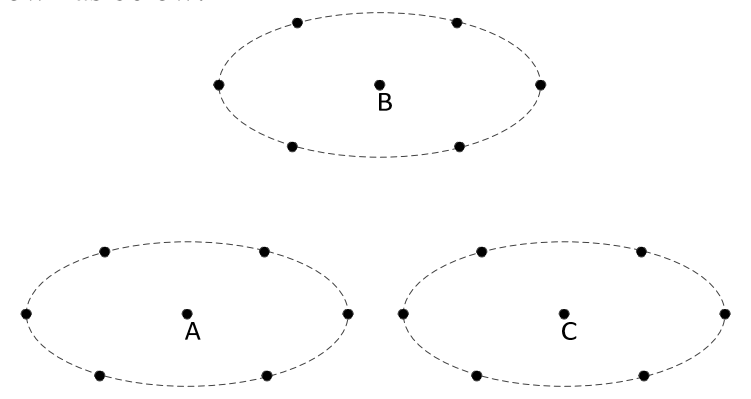

Figure 2 Anti-jamming attitude measurement antenna array

The antenna $\mathrm{ABC}$ are arranged in an equilateral triangle, each attitude measurement antenna is an array of 7 elements. The attitude is carried out by using the synthetic signal of each antenna array. The advantages of this array structure is that the attitude can be carried out in the interference condition, its disadvantage is the use of a large array of elements, but the carrier frequency of the navigation signal is higher, so the radius of each antijamming antenna is $r \leq \lambda / 2=9.52 \mathrm{~cm}$, the structure of the antenna can be used in many application scenarios.

\subsection{Double difference method for antenna attitude measurement}

The attitude is carried out by means of the double difference method, the attitude information of the carrier is measured by the data obtained from the three position measuring antenna.

We observe measurement antenna's output of $A$ and $B$ received from satellite $i, j$. The difference between the measured value of the carrier signal of the two measuring antenna for the same satellite is called the single difference, the difference between the two single difference measurements of satellite $i$ and $j$ is called the double difference. As shown in fig. 3: 


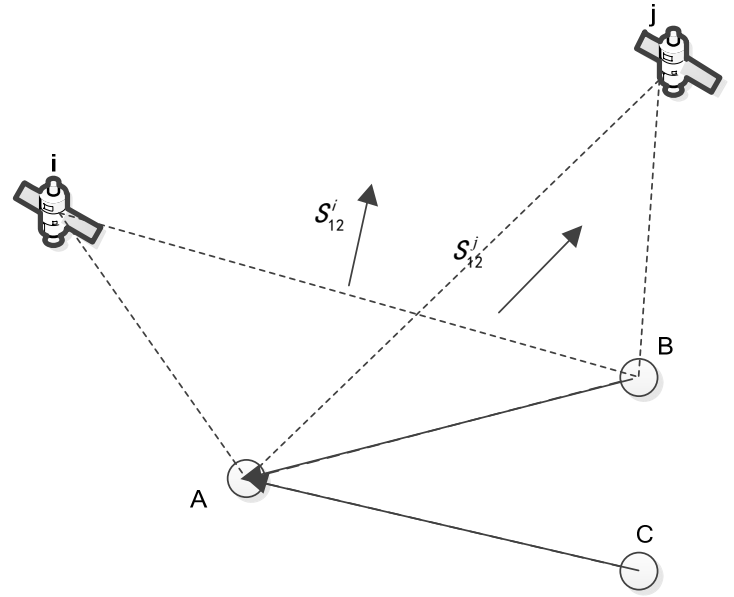

Figure 3 Schematic diagram of double difference method

The observation equation of the carrier phase for the double difference method is[6] (for the measurement antenna $\mathrm{AB})$ :

$$
\begin{aligned}
\Delta \Phi_{\mathrm{AB}}^{i j}= & \frac{1}{\lambda}\left(d_{\mathrm{AB}}^{i}+c \Delta \gamma_{m_{\mathrm{AB}}^{i}}\right)-N_{\mathrm{AB}}^{i}+\varepsilon_{\mathrm{AB}}^{i} \\
& -\frac{1}{\lambda}\left(d_{\mathrm{AB}}^{j}+c \Delta \gamma_{m_{\mathrm{AB}}^{j}}\right)+N_{\mathrm{AB}}^{j}-\varepsilon_{\mathrm{AB}}^{j} \\
= & \frac{1}{\lambda}\left(d_{\mathrm{AB}}^{i j}+c \Delta \gamma_{m_{\mathrm{AB}}^{i j}}\right)-N_{\mathrm{AB}}^{i j}+\varepsilon_{\mathrm{AB}}^{i j}
\end{aligned}
$$

where $\lambda$ is the signal wavelength, $c$ is the speed of light, $d_{\mathrm{AB}}^{i j}$ is the double difference of the distance between the satellite and the antenna, $\Delta \gamma_{m_{\mathrm{AB}}^{i j}}$ is the multipath error of the double difference, $N_{\mathrm{AB}}^{i j}$ is the double difference of the ambiguity, $\varepsilon_{\mathrm{AB}}^{i j}$ is the measurement error.

Combined with the antenna structure mentioned above, without considering the multipath error and measurement error, the double difference observation equation of carrier phase is:

$$
\Delta \Phi_{\mathrm{AB}}^{i j}=d_{\mathrm{AB}}^{i j} / \lambda-N_{\mathrm{AB}}^{i j}
$$

According to the double difference carrier phase observation equation of multi satellites, the integer ambiguity can be solved by the LAMBDA method. The attitude of baseline $\mathrm{AB}$ and baseline $\mathrm{AC}$ is calculated by the least square method. The information of the two baseline is calculated to get the attitude information of the carrier.

\section{Simulation results}

Based on LCMV algorithm, the adaptive nulling algorithm of 7 array elements circle array with core is simulated[7]. In the simulation, the number of jamming is 1 , the jamming direction is $(10 \pi / 9, \pi / 18)$. Simulation result is shown as Fig.4.

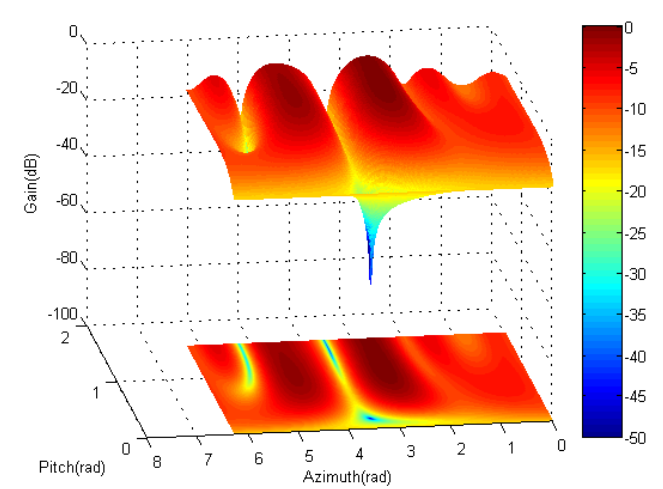

Figure 4 Pattern of adaptive nulling antennas

As can be seen in Fig.4, there is a nulling in the direction of interference whose depth is about $-45 \mathrm{~dB}$. Strong jamming can be suppressed by the adaptive nulling antenna. The effect of the attitude measurement algorithm is ensured.

When the jamming exists, the received GPS signal is interfered by the same frequency jamming, and the simulation results are shown below.
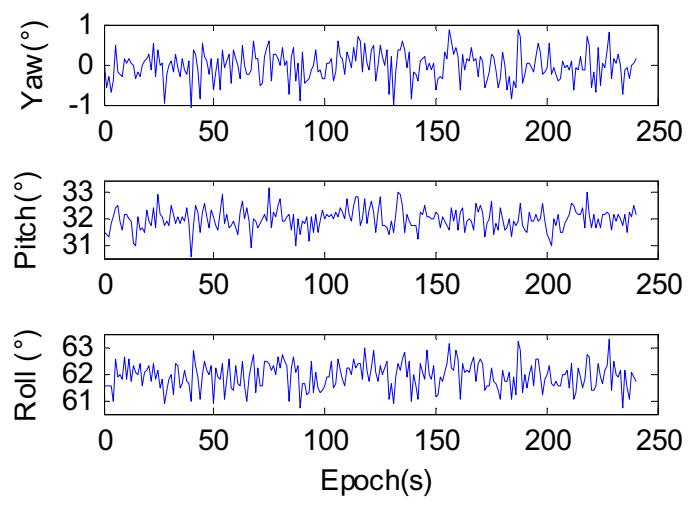

Figure 5 Attitude accuracy without anti-jamming algorithm

As can be seen from Fig.5, when there is no antijamming algorithm, attitude accuracy is reduced because of the presence of jamming. The accuracy is about 2 degrees.
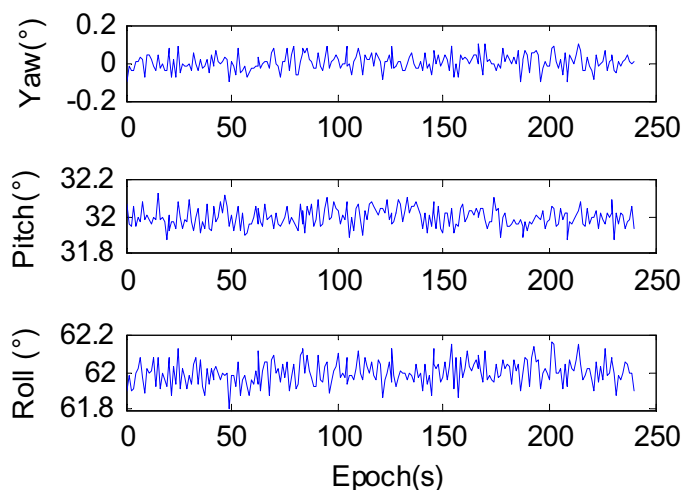

Figure 6 Attitude accuracy with anti-jamming algorithm

As we can be seen from Fig. 6, when using the nulling algorithm, the accuracy of the attitude measurement is about 0.4 degrees. This is much higher than that without the use of anti-jamming algorithm. and then verify the effectiveness of the attitude measurement algorithm using the zero modulation antenna. 
The simulation results verify the effectiveness of the zero nulling antenna to improve the accuracy of the attitude measurement algorithm.

\section{Conclusions}

We propose a kind of anti-jamming attitude measurement method. Firstly, the antenna array is constructed, which is composed of 3 anti-jamming antenna array, and the antenna array is a 7 element antenna array. Before the attitude determination is carried out, the direction of the jamming signal should be estimated by the MUSIC algorithm, then the antijamming reception of the navigation signal is realized by using the nulling algorithm. Finally, the attitude measurement is realized by the double difference method.

The simulation results show that the anti-jamming attitude measurement method can realize attitude measurement with high accuracy in the presence of strong jamming. Compared with the attitude measurement method without anti-jamming algorithm, it has higher practical value.

The disadvantage of this method is that it needs more computation in the process. The constraint method is also should be studied for the carrier phase without distortion, which is a direction that is worth improving.

\section{References}

1. Zhang, Jian Jun and J. H. Xiang. Study on Effect of Channel Uncertainties on GPS Antenna Adaptive Algorithm. Radio Engineering (2010).

2. Raasakka J and Orejas M. Analysis of notch filtering methods for narrowband interference mitigation. Position, Location and Navigation Symposium Plans 2014, 1282-1292( 2014).

3. Ardalan A and Rezvani M. An iterative method for attitude determination based on misaligned GNSS baselines. IEEE Transactions on Aerospace \& Electronic Systems, 51:97-107(2015).

4. Li Qiang et al. A Robust Anti-Jamming Navigation Receiver with Antenna Array and GPS/SINS. Communications Letters IEEE 18.3:467-470(2014).

5. Wang Dawei, et al. Attitude aided space-time multibeamformer anti-jamming approach for satellite navigation receiver.368-372(2014).

6. Henkel $\mathrm{P}$ and Iafrancesco M. Tightly coupled position and attitude determination with two lowcost GNSS receivers. Wireless Communications Systems, 2014 11th International Symposium on IEEE, 895-900(2014).

7. El Khatib, Alaa, K. Assaleh, and H. Mir. Space-Time Adaptive Processing Using Pattern Classification. IEEE Transactions on Signal Processing 63.3:766$779(2015)$. 